\title{
ON A CLASS OF SEMILINEAR ELLIPTIC EQUATIONS WITH BOUNDARY CONDITIONS AND POTENTIALS WHICH CHANGE SIGN
}

\author{
M. OUANAN AND A. TOUZANI
}

Received 19 May 2004

We study the existence of nontrivial solutions for the problem $\Delta u=u$, in a bounded smooth domain $\Omega \subset \mathbb{R}^{\mathbb{N}}$, with a semilinear boundary condition given by $\partial u / \partial v=\lambda u-$ $W(x) g(u)$, on the boundary of the domain, where $W$ is a potential changing sign, $g$ has a superlinear growth condition, and the parameter $\left.\lambda \in] 0, \lambda_{1}\right] ; \lambda_{1}$ is the first eigenvalue of the Steklov problem. The proofs are based on the variational and min-max methods.

\section{Introduction}

In this paper, we study the existence of nontrivial solutions of the following problem:

$\left(P_{\lambda}\right)$

$$
\begin{gathered}
\Delta u=u \quad \text { in } \Omega, \\
\frac{\partial u}{\partial \nu}=\lambda u-W(x) g(u) \quad \text { on } \partial \Omega,
\end{gathered}
$$

where $\Omega$ is a bounded domain set of $\mathbb{R}^{\mathbb{N}}, \mathbb{N} \geq 3$ with smooth boundary $\partial \Omega, \Delta u=\nabla \cdot(\nabla u)$ is the Laplacian and $\partial / \partial \nu$ is the outer normal derivative; the parameter $\left.\lambda \in] 0, \lambda_{1}\right]$, where $\lambda_{1}$ is the first eigenvalue of the Steklov problem (see [5]), $W \in C(\bar{\Omega})$ different from zero almost everywhere and changes sign, while $g(u)$ is a continuous and superlinear function (see $(G 1),(G 2),(G 3)$ ) below.

In the case of $W \equiv 0,\left(P_{\lambda}\right)$ becomes a linear eigenvalue problem and it is known as the Steklov problem studied in [5], which proved the existence, the simplicity, and the isolation of the first eigenvalue $\lambda_{1}$.

The study of the similar problem when the nonlinear term is placed in the equation, that is, when one considers problem of the form $-\Delta u=\lambda u+W(x) g(u)$ with Dirichlet boundary condition, there is more work; hence, in the case where $g$ behaves as a power near 0 and infinity, Alama and Tarantello in [2] showed the existence of a positive solution, provided that $f$ is odd, and found that a necessary and sufficient condition to obtain 
such a solution is

$$
\int_{\Omega} W(x) e_{1}^{p} d x<0
$$

where $e_{1}$ denotes a positive eigenfunction of Laplacian related to the first eigenvalue, with $p \in] 2,2^{*}\left[, 2^{*}=2 \mathbb{N} /(\mathbb{N}-2)\right.$ if $\mathbb{N}>2,2^{*}=+\infty$ if $\mathbb{N}=2$. Also, in [3], it was proved that (1.2) is a necessary and sufficient condition to obtain a positive solution; recently, Margone in [14], proved some results of existence in case that $0<\lambda \leq \lambda_{1}$, close to $\lambda_{1}$; by using mountain pass lemma (see [4]) and linking-type theorem (see [15]). Finally, in [1], Alama and Delpino proved under some restriction on the sign of $W(x)$ the existence of nontrivial solution, by using two different approach: one involving min-max methods, the other Morse theory methods.

However, nonlinear boundary conditions have only been considered in recent years, for the Laplacian with boundary conditions, see, for example $[6,7,8,12,13,16]$, where the authors discussed mountain pass theorem on an order interval with Dirichlet boundary condition. For elliptic systems with nonlinear boundary conditions, see $[9,10]$.

The main purpose of this work is to study one problem of Neumman boundary value, in the case $\lambda=\lambda_{1}$ because if $\lambda<\lambda_{1}$, it is easy to prove that the functional $\Phi_{\lambda}$ has a condition of mountain pass structure. We show two results of existence obtained as critical points of the functional related at $\left(P_{\lambda}\right)$, by using mountain pass lemma introduced in [4] and linking-type theorem introduced in [15].

The rest of this paper is organized as follows: in Section 2, we cite the main results and in Section 3, we prove the main results.

\section{Main results}

In the sequel, we consider the following functional:

$$
G(u)=\int_{0}^{u} g(t) d t
$$

Then, we show the following existence results for $\left(P_{\lambda}\right)$.

THeORem 2.1. Let $g$ be a continuous real-valued function on $\mathbb{R}$ such that the following assumptions hold:

(G1) $g(u) u \geq 0$ for all $u \in \mathbb{R}$,

(G2) $|g(u)| \leq C|u|^{r-1}$ for all $u \in \mathbb{R}$, and for some $\left.r \in\right] 2,2(\mathbb{N}-1) /(\mathbb{N}-2)[$,

(G3) $g(u) u \geq(s+1) G(u)$ for $u>R, R$ sufficiently large, and for some $s \in] 1, \mathbb{N} /(\mathbb{N}-2)[$,

(G4) $\lim _{u \rightarrow 0}\left(g(u) /|u|^{r-2} u\right)=a>0$,

(G5) $g(u) u \geq c|u|^{s+1}$ for $|u|>R$, and $R$ sufficiently large,

(G6) $W^{-}(g(u) u-(s+1) G(u)) \leq \gamma|u|^{2},|u|>R$, for some

$$
\gamma \in] 0,\left(\frac{s+1}{2}-1\right)\left(\lambda_{2}-\lambda_{1}\right)[
$$

where $\lambda_{2}$ is the second eigenvalue of the Steklov problem, and $W^{-}(x)=-\min \{W(x), 0\}$, $W^{-}=\max _{x \in \partial \Omega} W^{-}(x)$; moreover, let 
$\left(W_{0}\right) W^{+}(x)=\max \{W(x), 0\}, \operatorname{meas}(\{x \in \partial \Omega: W(x)=0\})=0$,

$\left(W_{1}\right) \int_{\partial \Omega} W(x) e_{1}^{r} d \sigma<0$, where $e_{1}$ is a positive eigenfunction related to $\lambda_{1}$, then $\left(P_{\lambda}\right)$ has a positive solution $u_{\lambda}$ for any $\lambda \in\left(0, \lambda_{1}\right]$.

Remarks 2.2. (i) Condition (G6) was introduced by Girardi and Matzeu (see [11]) and plays a crucial role in the proof of Palais-Smale condition.

(ii) Condition $\left(W_{1}\right)$ is necessary and sufficient to obtain such a solution and was introduced by Alama and Tarantello, (see [3]), for semilinear elliptic equations with Dirichlet boundary conditions.

Theorem 2.3. Let $g$ satisfy conditions (G1)-(G3), (G5), (G6), and $\left(W_{0}\right)$. If $W$ verifies the further assumptions,

$\left(W_{2}\right) \int_{\partial \Omega} W(x) G\left(t e_{1}\right) d \sigma>0$, for all $t \in \mathbb{R} \backslash\{0\}$,

$\left(W_{3}\right) \int_{D} W(x) G\left(t_{1}\right) d \sigma>c$, for all $t \in \mathbb{R}$ and for some $c \in \mathbb{R}$, where $D$ is a nonempty open subset in $\partial \Omega$ such that supp $W^{-} \subset D$,

then $\left(P_{\lambda_{1}}\right)$ has a nontrivial solution.

Remark 2.4. Note that the solution found in Theorem 2.3 is surely not always positive because $\left(W_{1}\right)$ does not hold. Moreover, condition $\left(W_{2}\right)$, which appears in Theorem 2.3, is in some sense complementary to $\left(W_{1}\right)$ if $g$ is a power.

\section{Proof of the main results}

It is well known that the solutions of $\left(P_{\lambda}\right)$ are critical points of the functional

$$
\Phi_{\lambda}(u)=\frac{1}{2}\left(\|\nabla u\|_{2}^{2}+\|u\|_{2}^{2}-\lambda \int_{\partial \Omega}|u|^{2} d \sigma\right)-\int_{\partial \Omega} W(x) G(u) d \sigma, \quad u \in H^{1}(\Omega) .
$$

In order to prove the main results, we apply the mountain pass theorem (see [4]) and a suitable version of the linking-type theorem (see [15]) to the functional $\Phi_{\lambda}$.

The following lemma is the key for proving our theorems, in which we consider $\lambda=\lambda_{1}$ because if $\lambda<\lambda_{1}$, the argument is the same.

Lemma 3.1. Under assumptions $\left(W_{0}\right),(G 2),(G 3),(G 5),(G 6)$, the functional $\Phi_{\lambda}(u)$ satisfies the Palais-Smale condition on $H^{1}(\Omega)$. That is, any sequence $\left(u_{n}\right)_{n}$ in $H^{1}(\Omega)$, such that

$$
\left(\Phi_{\lambda}\left(u_{n}\right)\right)_{n} \text { is bounded and } \Phi_{\lambda}^{\prime}\left(u_{n}\right) \longrightarrow 0
$$

possesses a converging subsequence.

Proof. Let $\left(u_{n}\right)_{n} \subset H^{1}(\Omega)$ be a Palais-Smale sequence, namely, there exist $c_{1}$ and $c_{2}$ such that

$$
\begin{aligned}
& c_{1} \leq \frac{1}{2}\left(\left\|\nabla u_{n}\right\|_{2}^{2}+\right.\left.\left\|u_{n}\right\|_{2}^{2}-\lambda_{1} \int_{\partial \Omega}\left|u_{n}\right|^{2} d \sigma\right)-\int_{\partial \Omega} W(x) G\left(u_{n}\right) d \sigma \leq c_{2}, \\
& \sup _{\left\{\phi \in H^{1}(\Omega),\|\phi\|_{1,2}=1\right\}}\left\{\int_{\Omega}\left(\nabla u_{n} \nabla \phi+u_{n} \phi\right) d x-\lambda_{1} \int_{\partial \Omega} u_{n} \phi d \sigma\right. \\
&\left.-\int_{\partial \Omega} W(x) g\left(u_{n}\right) \phi d \sigma\right\} \longrightarrow 0 \text { as } n \longrightarrow+\infty .
\end{aligned}
$$


We are going to show that $\left(u_{n}\right)_{n}$ is bounded in $H^{1}(\Omega)$. By assumptions $(G 3)$ and $(G 6)$, and from (3.3) and (3.4), we get for some constant $c_{R}>0$ depending on the number $R$ of (G3),

$$
\begin{aligned}
\int_{\Omega}\left(\left|\nabla u_{n}\right|^{2}+u_{n}^{2}\right) d x= & \lambda_{1} \int_{\partial \Omega} u_{n}^{2} d \sigma-\int_{\partial \Omega} W(x) g\left(u_{n}\right) u_{n} d \sigma+\epsilon_{n}\left\|u_{n}\right\|_{1,2} \\
\geq & \lambda_{1} \int_{\partial \Omega} u_{n}^{2} d \sigma+\int_{\partial \Omega} W^{+}(x) g\left(u_{n}\right) u_{n} d \sigma \\
& -\int_{\partial \Omega} W^{-}(x) g\left(u_{n}\right) u_{n} d \sigma+\epsilon_{n}\left\|u_{n}\right\|_{1,2} \\
\geq & \lambda_{1} \int_{\partial \Omega} u_{n}^{2} d \sigma+(s+1) \int_{\partial \Omega} W^{+}(x) G\left(u_{n}\right) d \sigma-\gamma \int_{\partial \Omega \cap\{|u|>R\}}\left|u_{n}\right|^{2} d \sigma \\
& -(s+1) \int_{\partial \Omega \cap\{|u|>R\}} W^{-}(x) G\left(u_{n}\right) d \sigma+c_{R}+\epsilon_{n}\left\|u_{n}\right\|_{1,2} \\
\geq & \lambda_{1} \int_{\partial \Omega} u_{n}^{2} d \sigma+(s+1)\left[\frac{1}{2}\left\|u_{n}\right\|_{1,2}^{2}-\frac{\lambda_{1}}{2} \int_{\partial \Omega} u_{n}^{2} d \sigma-c_{2}\right] \\
& -\gamma \int_{\partial \Omega} u_{n}^{2} d \sigma+c_{R}+\epsilon_{n}\left\|u_{n}\right\|_{1,2} .
\end{aligned}
$$

Set $X_{1}=\operatorname{vect}\left(e_{1}\right)$, then, there exist $k_{n} \in \mathbb{R}$ such that $u_{n}=k_{n} e_{1}+v_{n}$, where $v_{n} \in X_{1}^{\perp}$.

Using the variational characterization of $\lambda_{2}$, (3.5) becomes

$$
\left(\frac{s+1}{2}-1\right)\left(1-\frac{\lambda_{1}}{\lambda_{2}}\right)\left\|v_{n}\right\|_{1,2}^{2}+\epsilon_{n}\left\|v_{n}\right\|_{1,2} \leq \gamma \int_{\partial \Omega}\left(k_{n} e_{1}+v_{n}\right)^{2} d \sigma+c
$$

where $\epsilon_{n}$ is an infinitesimal sequence of positive numbers.

On the other hand, using variational characterization of $\lambda_{1}$, it follows that

$$
\left[\left(\frac{s+1}{2}-1\right)\left(1-\frac{\lambda_{1}}{\lambda_{2}}\right)-\frac{\gamma}{\lambda_{2}}\right]\left\|v_{n}\right\|_{1,2}^{2}+\epsilon_{n}\left\|v_{n}\right\|_{1,2} \leq c+\gamma k_{n}^{2} \int_{\partial \Omega} e_{1}^{2} d \sigma
$$

On the other side, by (2.2) and taking into acount that $\epsilon_{n} \rightarrow 0$, we deduce that

$$
\left\|v_{n}\right\|_{1,2}^{2} \leq \operatorname{const}\left(1+k_{n}^{2}\right)
$$

hence, it suffices to prove that $\left(\left|k_{n}\right|\right)_{n}$ is bounded. So, if $\left|k_{n}\right| \rightarrow+\infty$ (at least a subsequence), therefore $\left(v_{n} /\left|k_{n}\right|\right)_{n}$ is bounded in $H^{1}(\Omega)$, so a subsequence, also called $\left(v_{n} /\left|k_{n}\right|\right)_{n}$, weakly converges in $H^{1}(\Omega)$ at some $f$ and that

$$
f(x)+e_{1}(x) \neq 0 \quad \text { a.e. in } \bar{\Omega} \text {. }
$$

Indeed, if (3.9) is false, taking into acount that

$$
\int_{\Omega}\left(\nabla\left(\frac{v_{n}}{\left|k_{n}\right|}\right) \nabla e_{1}+\frac{v_{n}}{\left|k_{n}\right|} e_{1}\right) d x=0 \quad \forall n \in \mathbb{N}
$$


as $n \rightarrow+\infty$, we obtain $\left\|e_{1}\right\|_{1,2}^{2}=\lambda_{1} \int_{\partial \Omega} e_{1}^{2}=0$, which is an absurdum as we know that $e_{1}$ is the principal eigenvector related with $\lambda_{1}$.

From (3.4), we obtain

$$
\int_{\Omega}\left(\nabla u_{n} \nabla \phi+u_{n} \phi\right) d x-\lambda_{1} \int_{\partial \Omega} u_{n} \phi d \sigma-\int_{\partial \Omega} W(x) g\left(u_{n}\right) \phi d \sigma=\eta_{n}
$$

with $\lim _{n \rightarrow+\infty} \eta_{n}=0$ in $\mathbb{R}$.

Let $\phi_{n}=\left(k_{n} e_{1}+v_{n}\right)\left|k_{n}\right|^{-1} \phi$, where $\phi$ is a regular function with support compact in $\bar{\Omega}$ and meas $(\operatorname{supp} \phi \cap \partial \Omega) \neq 0$; then

$$
\begin{aligned}
\int_{\Omega}( & \left.\nabla\left(k_{n} e_{1}+v_{n}\right) \nabla \phi_{n}+\left(k_{n} e_{1}+v_{n}\right) \phi_{n}\right) d x \\
& -\lambda_{1} \int_{\partial \Omega}\left(k_{n} e_{1}+v_{n}\right) \phi_{n} d \sigma-\int_{\partial \Omega} W(x) g\left(k_{n} e_{1}+v_{n}\right) \phi_{n} d \sigma=\eta_{n},
\end{aligned}
$$

hence

$$
\begin{aligned}
\frac{1}{\left|k_{n}\right|} \int_{\Omega}[ & \left.\nabla v_{n} \nabla \phi_{n}+v_{n} \phi_{n}\right] d x-\frac{\lambda_{1}}{\left|k_{n}\right|} \int_{\partial \Omega} v_{n} \phi_{n} d \sigma \\
= & \frac{1}{\left|k_{n}\right|} \int_{\partial \Omega} W(x) g\left(k_{n} e_{1}+v_{n}\right) \phi_{n} d \sigma+o(1)
\end{aligned}
$$

for $n$ large enough.

So, Hölder inequality and (3.8) imply that $\left(1 /\left|k_{n}\right|\right) \int_{\Omega}\left(\nabla v_{n} \nabla \phi_{n}+v_{n} \phi_{n}\right) d x$ and $\left(\lambda_{1} /\right.$ $\left.\left|k_{n}\right|\right) \int_{\partial \Omega} v_{n} \phi_{n} d \sigma$ are bounded.

On the other side, combining $\left(W_{0}\right)$ and (3.9), it follows that either

$$
\int_{\text {Supp } W^{+}}\left|h(x)+e_{1}(x)\right|^{s+1} d \sigma>0 \quad \text { or } \quad \int_{\text {Supp } W^{-}}\left|h(x)+e_{1}(x)\right|^{s+1} d \sigma>0 .
$$

In the first case, we take $\phi$ regular nonnegative function with meas $\left(\operatorname{supp} \phi \cap \operatorname{supp} W^{+}\right) \neq 0$ such that

$$
\int_{\text {Supp } W^{+}} W^{+}(x) \phi(x)\left|h(x)+e_{1}(x)\right|^{s+1} d \sigma>0
$$

then, by (G6) and (3.15), we get for some positive constant $c$,

$$
\begin{aligned}
\frac{1}{\left|k_{n}\right|} \int_{\partial \Omega} W(x) g\left(k_{n} e_{1}+v_{n}\right) \phi_{n} d \sigma & \geq \frac{c}{\left|k_{n}\right|^{2}} \int_{\operatorname{supp} W^{+}} W^{+}(x)\left|k_{n} e_{1}+v_{n}\right|^{s+1} \phi d \sigma-c \\
& \geq c k_{n}^{s-1} \int_{\operatorname{supp} W^{+}} W^{+}(x)\left|e_{1}+\frac{v_{n}}{k_{n}}\right|^{s+1} \phi d \sigma-c \longrightarrow+\infty
\end{aligned}
$$

This and formula (3.13) contradict the bound of $\left(1 /\left|k_{n}\right|\right) \int_{\Omega}\left(\nabla v_{n} \nabla \phi_{n}+v_{n} \phi_{n}\right) d \sigma$ and $\left(\lambda_{1} /\left|k_{n}\right|\right) \int_{\partial \Omega} v_{n} \phi_{n} d \sigma$. 
For the second case, it suffices to take $\phi$ nonnegative function with meas $(\operatorname{supp} \phi \cap$ $\left.\operatorname{supp} W^{-}\right) \neq 0$ such that

$$
\int_{\text {Supp } W^{-}} W^{-}(x) \phi(x)\left|h(x)+e_{1}(x)\right|^{s+1} d \sigma>0 .
$$

Finally, we have proved that $\left(u_{n}\right)_{n}$ is bounded, this implies the existence of a subsequence weakly converging in $H^{1}(\Omega)$. On the other side, thanks to $(G 2)$ and the compact embed$\operatorname{ding} H^{1}(\Omega) \hookrightarrow L^{r}(\partial \Omega)$ for $\left.r \in\right] 2,2(N-1) /(\mathbb{N}-2)$ [, we have the strong convergence.

LEMma 3.2. The origin is a strict locale minimizer of $\Phi_{\lambda}$.

Proof. First, remark that each $u \in H^{1}(\Omega)$ can be written as $u=t e_{1}+v$, where $t \in \mathbb{R}$, and $v \in X_{1}^{\perp}$, then

$$
\int_{\Omega}\left(|\nabla u|^{2}+|u|^{2}\right) d x=t^{2} \lambda_{1} \int_{\partial \Omega} e_{1}^{2} d \sigma+\|v\|_{1,2}^{2}
$$

Choosing $e_{1}$ such that $\int_{\partial \Omega} e_{1}^{2} d \sigma=1 / \lambda_{1}$, one gets, for all $u$ satisfying $\|u\|_{1,2} \leq 1 / 2\left\|e_{1}\right\|_{\infty}$,

$$
t^{2}<\|u\|_{1,2}^{2}<\frac{1}{4\left\|e_{1}\right\|_{\infty}^{2}}
$$

Hence, by variational characterization of the eigenvalues of the Laplacian with boundary conditions and for a suitable function $F(t, v)$, we obtain

$$
\begin{aligned}
\Phi_{\lambda_{1}}(u) & \geq \frac{1}{2}\left(1-\frac{\lambda_{1}}{\lambda_{2}}\right)\|v\|_{1,2}^{2}-\int_{\partial \Omega} W(x) G\left(t e_{1}+v\right) d \sigma \\
& \geq \frac{1}{2}\left(1-\frac{\lambda_{1}}{\lambda_{2}}\right)\|v\|_{1,2}^{2}-|t|^{r} \int_{\partial \Omega} W(x) e_{1}^{r} d \sigma+F(t, v),
\end{aligned}
$$

where by (G4),

$$
\begin{aligned}
F(t, v) & =\int_{\partial \Omega} W(x)\left[\left|t e_{1}\right|^{r}-G\left(t e_{1}\right)\right] d \sigma+\int_{\partial \Omega} W(x)\left[G\left(t e_{1}\right)-G\left(t e_{1}+v\right)\right] d \sigma \\
& =\int_{\partial \Omega} W(x)\left[G\left(t e_{1}\right)-G\left(t e_{1}+v\right)\right] d \sigma+o\left(|t|^{r}\right) .
\end{aligned}
$$

On the other hand, using arrangement-finite theorem, there exists a function $0<\theta \equiv$ $\theta(x, t, v)<1$ such that

$$
\left|G\left(t e_{1}+v\right)-G\left(t e_{1}\right)\right|=\left|g\left(t e_{1}+\theta v(x)\right) v(x)\right|
$$


In case that $\left|t e_{1}+\theta v(x)\right| \geq 1$, by (3.19), we deduce

$$
|\theta v(x)| \geq 2|t|\left\|e_{1}\right\|_{\infty}-|t|\left\|e_{1}\right\|_{\infty} \geq|t|\left\|e_{1}\right\|_{\infty},
$$

so by (G2),

$$
\begin{aligned}
\left|g\left(t e_{1}+\theta v(x)\right) v(x)\right| & \leq C\left|t e_{1}+\theta v(x)\right|^{r-1}|v(x)| \\
& \leq 2^{r-2} C|\theta v(x)|^{r-1}|v(x)| \leq 2^{r-1} C|v(x)|^{r},
\end{aligned}
$$

while, if $\left|t e_{1}+\theta v(x)\right| \leq 1$, using again (G2), one obtains

$$
\begin{aligned}
& |W(x)|\left|g\left(t e_{1}+\theta v(x)\right) v(x)\right| \leq C\left|t e_{1}+\theta v(x)\right|^{r-1} v(x) \\
& \leq C\left[\left|t e_{1}\right|^{r-1}+|v(x)|^{r}\right] \leq \epsilon\left|t e_{1}\right|^{r}+C_{\epsilon}|v(x)|^{r},
\end{aligned}
$$

where $\epsilon, C_{\epsilon}$ are two positive constants.

Set $A=-\int_{\partial \Omega} W(x) e_{1}^{r} d \sigma>0$. Combining (3.21), (3.24), and (3.25), and using $\left(W_{1}\right)$, (3.20) becomes

$$
\begin{aligned}
\Phi_{\lambda_{1}}(u) \geq & \frac{1}{2}\left(1-\frac{\lambda_{1}}{\lambda_{2}}\right)\|v\|_{1,2}^{2}-t^{r} \int_{\partial \Omega} W(x) e_{1}^{r}-|F(t, v)| \\
\geq & \frac{1}{2}\left(1-\frac{\lambda_{1}}{\lambda_{2}}\right)\|v\|_{1,2}^{2}+t^{r} A-2^{r-1} C \int_{\partial \Omega \cap\{|u|>1\}}|W(x)||v(x)|^{r} d \sigma \\
& -\int_{\partial \Omega \cap\{|u| \leq 1\}}\left[\epsilon\left|t e_{1}\right|^{r}+C_{\epsilon}|v(x)|^{r}\right]+\theta\left(|t|^{r}\right) \\
\geq & \frac{1}{2}\left(1-\frac{\lambda_{1}}{\lambda_{2}}\right)\|v\|_{1,2}^{2}+t^{r}\left(A-C_{1} \epsilon\right)-C_{2}\|v\|_{r}^{r}+o\left(|t|^{r}\right),
\end{aligned}
$$

where $C_{1}, C_{2}$ are two positive constants.

Hence, using Sobolev trace embedding, for $\epsilon<A / C_{1}$, we deduce

$$
\Phi_{\lambda_{1}}(u) \geq \frac{1}{2}\left(1-\frac{\lambda_{1}}{\lambda_{2}}\right)\|v\|_{1,2}^{2}+C_{3} t^{r}-C_{4}\|v\|_{1,2}^{r}+o\left(|t|^{r}\right) .
$$

For $r>2$, the least expression is strictly positive as $\|v\|_{1,2}$ is close to 0 .

Proof of Theorem 2.1. We will study only the case $\lambda=\lambda_{1}$ because if $\lambda<\lambda_{1}$, it is easily proved that the functional $\Phi_{\lambda}$ has a condition of mountain pass structure.

Now, it suffices to prove that there exist $\bar{u} \in H^{1}(\Omega)$ such that $\|\bar{u}\|_{1,2}>\rho, \rho$ large enough satisfying $\Phi_{\lambda}(\bar{u})<0$ which completes the proof of Theorem 2.3.

Let $t \in \mathbb{R}$ and $\phi \in C_{0}^{\infty}\left(\operatorname{supp} W^{+}\right)$, where $W^{+}(x)=\max (W(x), 0)$ (note that $\phi$ is well defined, thanks to $\left.\left(W_{0}\right)\right)$.

Using (G4), we obtain

$$
\begin{aligned}
\Phi_{\lambda_{1}}(t \phi) & =\frac{t^{2}}{2}\left(\|\phi\|_{1,2}^{2}-\lambda_{1} \int_{\partial \Omega} \phi^{2} d \sigma\right)-\int_{\partial \Omega} W(x) G(t \phi) d \sigma \\
& \leq \frac{t^{2}}{2}\|\phi\|_{1,2}^{2}-C t^{r} \int_{\operatorname{supp} W^{+}} W^{+}(x)|\phi|^{r} d \sigma \longrightarrow-\infty \quad \text { as } t \longrightarrow+\infty .
\end{aligned}
$$


Then, there exists $t_{0}>0$ large enough, such that $\bar{u}=t_{0} \phi$. Hence, using mountain pass lemma, there exists a critical point $u$ of $\Phi_{\lambda_{1}}$ at the level

$$
c=\inf _{\gamma \in \Gamma} \max _{v \in \gamma([0,1])} \Phi_{\lambda_{1}}(v)>0,
$$

where $\Gamma=\left\{\gamma \in C\left([0,1], H^{1}(\Omega)\right): \gamma(0)=0, \gamma(\bar{u})=1\right\}$ is the class of the path joining the origin to $\bar{u}$.

The positivity of $u$ can be checked by a standard argument based on (3.29) (which yields the nonnegativity of $u$ ) and by the strong maximum principle of Vazquez [17] (which yields the strict positivity of $u$ ).

The proof of Theorem 2.3 is based on Lemma 3.1 and the following version of the linking theorem, see [15].

Proposition 3.3. Let $E$ be a real Banach space with $E=X_{1} \oplus X_{2}$, where $X_{1}$ is finite dimensional. Suppose $J \in C^{1}(E, \mathbb{R})$ satisfies the Palais-Smale condition and

(J1) there are two constants $\rho, \alpha>0$ such that $J(u) \geq \alpha$, for all $u \in X_{2}:\|u\|_{E}=\rho$,

(J2) there exists $\bar{x} \in X_{2}$ with $\|\bar{x}\|=1$ and $R>\rho$ such that, if

$$
Q=\left\{u \in E: u=w+\delta \bar{x} \text { with } w \in X_{1},\|w\| \leq R, \delta \in(0, R)\right\}
$$

then $J_{\mid \partial Q} \leq 0$.

Then J possesses a critical value $c \geq \alpha$.

Proof of Theorem 2.3. Set $E=H^{1}(\Omega)$ and $J=\Phi_{\lambda}$ in Proposition 3.3.

First, thanks to Lemma 3.1, $\Phi_{\lambda}$ satisfies Palais-Smale condition.

We take $X_{1}=\left\{t e_{1} / t \in \mathbb{R}\right\}$, then $X_{2}=\left\{v \in H^{1}(\Omega) / \int_{\Omega} v e_{1} d x=0\right\}$ and let $v \in X_{2},\|v\|_{1,2}=$ $\rho$, then

$$
\begin{aligned}
\Phi_{\lambda_{1}}(v) & =\frac{1}{2} \int_{\Omega}\left(|\nabla v|^{2}+|v|^{2}\right) d x-\frac{\lambda_{1}}{2} \int_{\partial \Omega} v^{2} d \sigma-\int_{\partial \Omega} W(x) G(u) d \sigma \\
& \geq \frac{1}{2}\left(1-\frac{\lambda_{1}}{\lambda_{2}}\right)\|v\|_{1,2}^{2}-C \sup _{\partial \Omega} W(x) \int_{\partial \Omega}|v|^{r} d \sigma \\
& \geq \frac{1}{2}\left(1-\frac{\lambda_{1}}{\lambda_{2}}\right) \rho^{2}-C \rho^{r} .
\end{aligned}
$$

Then, for $\rho$ small enough, we have $\Phi_{\lambda_{1}}(v) \geq \alpha$, so $(J 1)$ is verified.

As for the proof of (J2), first of all, we note that, as also observed in [15], it is enough to prove the following two properties:

(a) $\Phi_{\lambda_{1}}\left(t e_{1}\right) \leq 0$ for all $t \in \mathbb{R}$;

(b) there exist $\bar{v} \in X_{2} \backslash\{0\}$ and $\rho_{0}>\rho$ such that $\Phi_{\lambda_{1}}(u) \leq 0$ for all $u \in X_{1} \oplus[\bar{v}]$ and $|u| \geq \rho_{0}$.

For (a), we have

$$
\Phi_{\lambda_{1}}\left(t e_{1}\right)=-\int_{\partial \Omega} W(x) G\left(t e_{1}\right)
$$

which is not positive by $\left(W_{2}\right)$, and (a) follows. 
On the other side, let $\bar{v}$ be a sufficiently regular function in $X_{2} \backslash\{0\}$ such that supp $\bar{v} \subset$ $\bar{\Omega} \backslash D$ and meas $(\operatorname{supp} \bar{v} \cap \partial \Omega) \neq 0$, Hence, for $u \in X_{1} \oplus[\bar{v}]=\left\{t e_{1}+\delta \bar{v},(t, \delta) \in \mathbb{R}^{2}\right\}$, we obtain

$$
\begin{aligned}
\Phi_{\lambda_{1}}(u) & =\frac{\delta^{2}}{2}\left[\int_{\Omega}\left(|\nabla \bar{v}|^{2}+|\bar{v}|^{2}\right) d x-\lambda_{1} \int_{\partial \Omega}|\bar{v}|^{2} d \sigma\right]-\int_{\partial \Omega} W(x) G\left(t e_{1}+\delta \bar{v}\right) d \sigma \\
& \leq \frac{\delta^{2}}{2} \int_{\Omega}\left(|\nabla \bar{v}|^{2}+|\bar{v}|^{2}\right) d x-\int_{\partial \Omega \backslash D} W^{+}(x) G\left(t e_{1}+\delta \bar{v}\right) d \sigma-\int_{D} W(x) G\left(t e_{1}\right) d \sigma+c
\end{aligned}
$$

therefore, by $\left(W_{3}\right)$, one gets

$$
\Phi_{\lambda_{1}}\left(t e_{1}+\delta \bar{v}\right) \leq c\left(t^{2}+\delta^{2}\right)-c \int_{\partial \Omega \backslash D} W^{+}(x)\left|t e_{1}+\delta \bar{v}\right|^{s+1} d \sigma+c .
$$

We observe now that the map

$$
t e_{1}+\delta \bar{v} \in X_{1} \oplus[\bar{v}] \longrightarrow(t, \delta) \in \mathbb{R}^{2}
$$

is an isomorphism and that

$$
t e_{1}+\delta \bar{v} \longrightarrow\left(\int_{\partial \Omega \backslash D} W^{+}(x)\left|t e_{1}+\delta \bar{v}\right|^{s+1} d \sigma\right)^{1 /(s+1)}
$$

yields a norm from $X_{1} \oplus[\bar{v}]$ as it easily can be deduced from the fact that $-t e_{1}(x) \neq \delta \bar{v}(x)$ in $\bar{\Omega} \backslash D$ if $\delta^{2}+t^{2} \neq 0$ (indeed $e_{1}(x)>0$ everywhere on $\bar{\Omega}$, while $\bar{v}$ has a compact support in $\bar{\Omega} \backslash D$ ) therefore, as all the norms are equivalents in a finite dimensional space, we get, for some positive constant $c$,

$$
\Phi_{\lambda_{1}}\left(t e_{1}+\delta \bar{v}\right) \leq c\left(t^{2}+\delta^{2}\right)-c\left(t^{s+1}+\delta^{s+1}\right)+c
$$

then,

$$
\lim _{t^{2}+\delta^{2} \rightarrow+\infty} \Phi_{\lambda_{1}}\left(t e_{1}+\delta \bar{v}\right)=-\infty
$$

hence, $\Phi_{\lambda}$ satisfies the assumptions of Proposition 3.3, which completes the proof of Theorem 2.3.

\section{References}

[1] S. Alama and M. Del Pino, Solutions of elliptic equations with indefinite nonlinearities via Morse theory and linking, Ann. Inst. H. Poincaré Anal. Non Linéaire 13 (1996), no. 1, 95-115.

[2] S. Alama and G. Tarantello, Elliptic problems with nonlinearities indefinite in sign, J. Funct. Anal. 141 (1996), no. 1, 159-215.

[3] On semilinear elliptic equations with indefinite nonlinearities, Calc. Var. Partial Differential Equations 1 (1993), no. 4, 439-475.

[4] A. Ambrosetti and P. H. Rabinowitz, Dual variational methods in critical point theory and applications, J. Funct. Anal. 14 (1973), no. 4, 349-381.

[5] I. Babuška and J. Osborn, Eigenvalue problems, Handbook of Numerical Analysis, Vol. II, Handb. Numer. Anal., II, North-Holland, Amsterdam, 1991, pp. 641-787. 


\section{Semilinear elliptic equations}

[6] M. Chipot, M. Chlebík, M. Fila, and I. Shafrir, Existence of positive solutions of a semilinear elliptic equation in $\mathbb{R}_{+}^{n}$ with a nonlinear boundary condition, J. Math. Anal. Appl. 223 (1998), no. $2,429-471$.

[7] M. Chipot, I. Shafrir, and M. Fila, On the solutions to some elliptic equations with nonlinear Neumann boundary conditions, Adv. Differential Equations 1 (1996), no. 1, 91-110.

[8] M. Del Pino and C. Flores, Asymptotic behavior of best constants and extremals for trace embeddings in expanding domains, Comm. Partial Differential Equations 26 (2001), no. 11-12, 2189-2210.

[9] J. Fernández Bonder, J. P. Pinasco, and J. D. Rossi, Existence results for Hamiltonian elliptic systems with nonlinear boundary conditions, Electron. J. Differential Equations 1999 (1999), no. $40,1-15$.

[10] J. Fernández Bonder and J. D. Rossi, Existence for an elliptic system with nonlinear boundary conditions via fixed-point methods, Adv. Differential Equations 6 (2001), no. 1, 1-20.

[11] M. Girardi and M. Matzeu, Existence and multiplicity results for periodic solutions of superquadratic Hamiltonian systems where the potential changes sign, NoDEA Nonlinear Differential Equations Appl. 2 (1995), no. 1, 35-61.

[12] B. Hu, Nonexistence of a positive solution of the Laplace equation with a nonlinear boundary condition, Differential Integral Equations 7 (1994), no. 2, 301-313.

[13] S. Li and Z.-Q. Wang, Mountain pass theorem in order intervals and multiple solutions for semilinear elliptic Dirichlet problems, J. Anal. Math. 81 (2000), 373-396.

[14] P. Magrone, On a class of semilinear elliptic equations with potential changing sign, Dynam. Systems Appl. 9 (2000), no. 4, 459-467.

[15] P. H. Rabinowitz, Some critical point theorems and applications to semilinear elliptic partial differential equations, Ann. Scuola Norm. Sup. Pisa Cl. Sci. (4) 5 (1978), no. 1, 215-223.

[16] S. Terracini, Symmetry properties of positive solutions to some elliptic equations with nonlinear boundary conditions, Differential Integral Equations 8 (1995), no. 8, 1911-1922.

[17] J. L. Vázquez, A strong maximum principle for some quasilinear elliptic equations, Appl. Math. Optim. 12 (1984), no. 3, 191-202.

M. Ouanan: Department of Mathematics and Informatics, Faculty of Sciences Dhar-Mahraz, P.O. Box 1796 Atlas-Fez, Fez, Morocco

E-mail address: m_ouanan@hotmail.com

A. Touzani: Department of Mathematics and Informatics, Faculty of Sciences Dhar-Mahraz, P.O. Box 1796 Atlas-Fez, Fez, Morocco

E-mail address: atouzani@menara.ma 


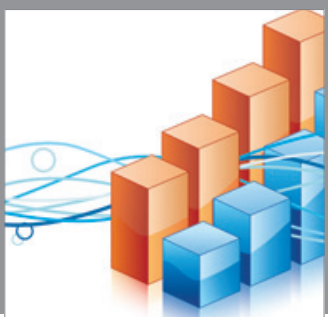

Advances in

Operations Research

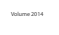

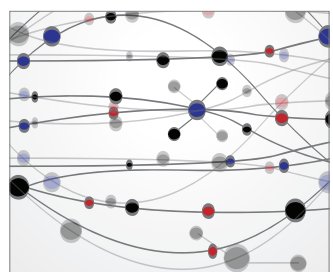

\section{The Scientific} World Journal
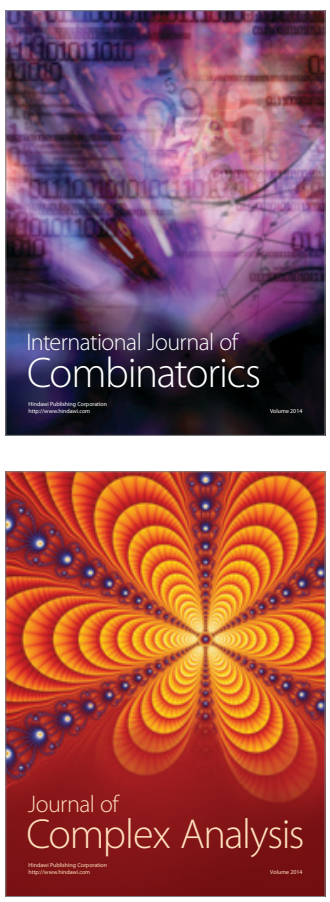

International Journal of

Mathematics and

Mathematical

Sciences
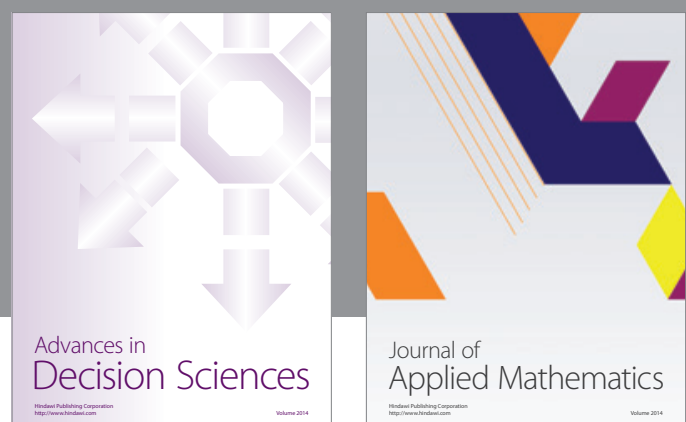

Journal of

Applied Mathematics
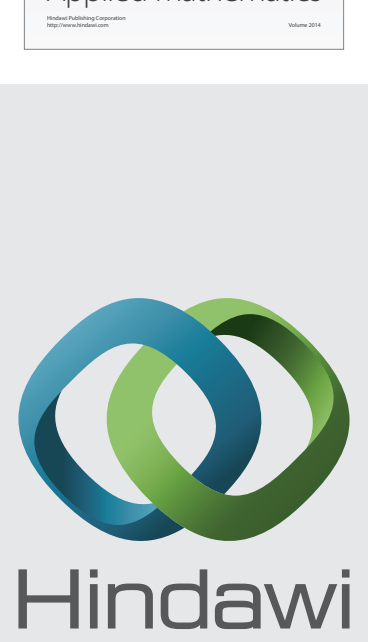

Submit your manuscripts at http://www.hindawi.com
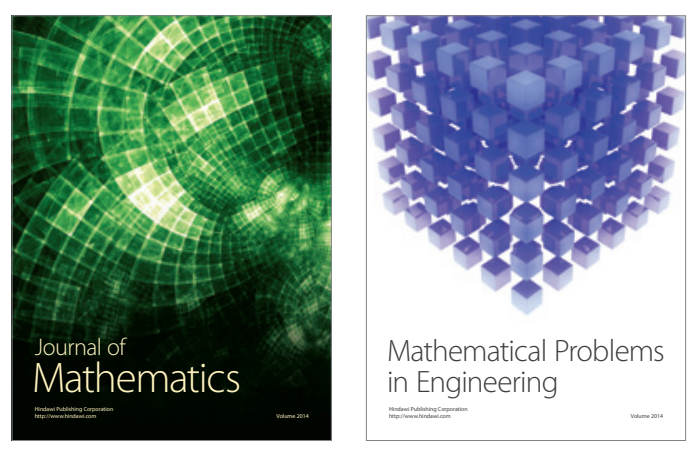

Mathematical Problems in Engineering
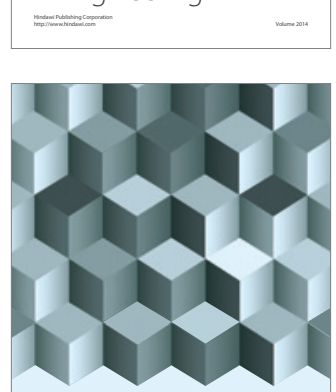

Journal of

Function Spaces
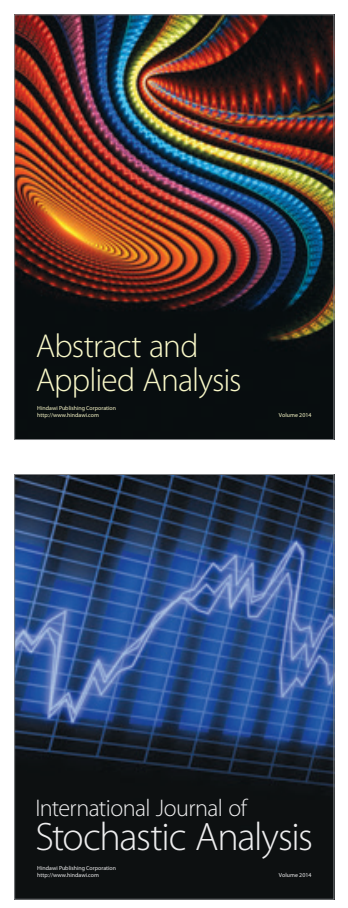

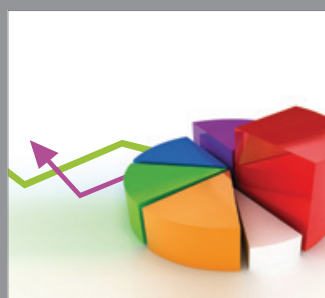

ournal of

Probability and Statistics

Promensencen
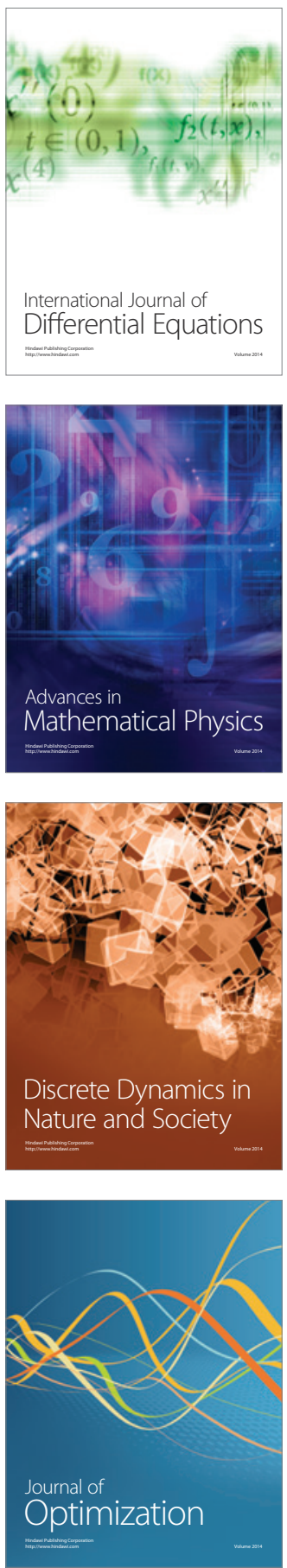